\title{
On-line Covering the Unit Square with Squares
}

\author{
by \\ Janusz JANUSZEWSKI \\ Presented by Aleksander PEŁCZYŃSKI
}

Summary. The unit square can be on-line covered with any sequence of squares whose total area is not smaller than 4 .

1. Introduction. Let $C, C_{1}, C_{2}, \ldots$ be planar convex bodies. We say that the sequence $\left(C_{i}\right)$ permits a covering [a translative covering] of $C$ if there exist rigid motions [translations, respectively] $\sigma_{i}$ such that $C \subseteq \bigcup \sigma_{i} C_{i}$. The on-line covering is a covering in which we are given $C_{i}$, where $i>1$, only after the motion $\sigma_{i-1}$ has been provided; at the beginning we are given $C_{1}$. The on-line restriction means that each set $C_{i}$ must be assigned its place before the next one appears, and that the placement cannot be modified afterwards. The area of $C$ is denoted by $|C|$.

Denote by $v(C)$ the least number such that any (finite or infinite) sequence of positive homothetic copies of $C$ with total area greater than $v(C)|C|$ permits an on-line translative covering of $C$. Let

$$
I=\{(x, y) ; 0 \leq x \leq 1,0 \leq y \leq 1\} .
$$

Moon and Moser showed in [8] that any sequence of homothetic copies of $I$ with total area not smaller than 3 permits a translative (off-line) covering of $I$ (see also the survey paper [1]). The bound of 3 cannot be reduced; three squares of side length smaller than 1 , each with a side parallel to a side of $I$, cannot translatively cover $I$. Consequently, also $v(I) \geq 3$. Thus far it is still unknown whether or not $v(I)>3$. The first upper bound presented by Kuperberg in $[6]$ is that $v(I) \leq 16$. According to the method of the current bottom [3] we have $v(I) \leq 8$. By using the method of the $n$th segment [5] we obtain $v(I) \leq 389 / 60 \approx 6.483$. In [4] the method of the current bottom and 
top is presented. According to that method we have $v(I) \leq \frac{7}{4} \sqrt[3]{9}+\frac{13}{8} \approx 5.265$. The aim of this paper is to show that $v(I) \leq 4$. As a corollary, we show that $v(C) \leq 14$ for any planar convex body $C$.

A survey of results concerning on-line packings and coverings is given in [7].

2. The method of the movable bottom. Let $S_{1}, S_{2}, \ldots$ be a sequence of squares. Denote by $s_{i}$ the side length of $S_{i}$ and assume that a side of $S_{i}$ is parallel to a side of $I$ for $i=1,2, \ldots$ We describe an on-line method of translative covering of $I$ with $S_{1}, S_{2}, \ldots$. This method is a small modification of the method of the current bottom presented in [3].

If $s_{i}<1$, then denote by $d_{i}$ the number from the set $\left\{\frac{1}{2}, \frac{1}{4}, \frac{1}{8}, \ldots\right\}$ such that

$$
d_{i} \leq s_{i}<2 d_{i} .
$$

Let $R_{i} \subset S_{i}$ be a rectangle of width $d_{i}$ and height $s_{i}$.

If $s_{1} \geq 1$, then we cover $I$ with $S_{1}$ and we stop the covering process. Otherwise, $S_{1} \supset R_{1}$ is placed so that

$$
\sigma_{1} R_{1}=\left\{(x, y) ; 0 \leq x \leq d_{1}, 0 \leq y \leq s_{1}\right\} .
$$

By the part of $S_{1}$ used for the covering we mean $Q_{1}=\sigma_{1} R_{1}$.

Assume that $i>1$ and that the translations $\sigma_{1}, \ldots, \sigma_{i-1}$ have been provided. If $s_{i} \geq 1$, then we cover $I$ with $S_{i}$ and stop. Otherwise, we find the greatest number $b_{i} \leq 1$ such that each point of $I$ whose $y$-coordinate is smaller than $b_{i}$ has been covered with a rectangle $\sigma_{j} R_{j}$ with $j<i$. The set of points of $I$ with $y$-coordinate $b_{i}$ is called the $i$ th bottom. A point of the $i$ th bottom is a surface point if no point of $I$ with the same $x$-coordinate and with a larger $y$-coordinate has been covered yet. We place $S_{i} \supset R_{i}$ so that $\sigma_{i} R_{i}$ contains a surface point and that $\sigma_{i} R_{i}$ has the form

$$
\sigma_{i} R_{i}=\left\{(x, y) ; k d_{i} \leq x \leq(k+1) d_{i}, b_{i} \leq y \leq b_{i}+s_{i}\right\},
$$

where $k \in\left\{0,1, \ldots, d_{i}^{-1}-1\right\}$. The part of $S_{i}$ used for the covering is defined as

$$
Q_{i}=\sigma_{i} R_{i} \backslash\left(\sigma_{1} R_{1} \cup \cdots \cup \sigma_{i-1} R_{i-1}\right) .
$$

We stop when $b_{i}=1$ for an integer $i$; then $I$ has been covered with $\sigma_{1} R_{1}, \ldots$, $\sigma_{i-1} R_{i-1}$. This method is called the method of the movable bottom.

Lemma 1. Let $S_{i}$ be a square of side length smaller than 1 placed by the method of the movable bottom. The area of the part of $S_{i}$ used for the covering exceeds $\frac{1}{3}\left|S_{i}\right|$.

Proof. The part of $\sigma_{i} R_{i}$ covered by $\sigma_{1} R_{1}, \ldots, \sigma_{i-1} R_{i-1}$ is of area smaller than

$$
\frac{1}{2} d_{i} \cdot d_{i}+\frac{1}{4} d_{i} \cdot \frac{1}{2} d_{i}+\frac{1}{8} d_{i} \cdot \frac{1}{4} d_{i}+\cdots=\frac{2}{3} d_{i}^{2} .
$$


As a consequence, $\left|Q_{i}\right|>\left|R_{i}\right|-\frac{2}{3} d_{i}^{2}=s_{i} d_{i}-\frac{2}{3} d_{i}^{2}$. Since

$$
\begin{gathered}
\frac{1}{3} d_{i}^{2}-d_{i} d_{i}+\frac{2}{3} d_{i}^{2}=0, \\
\frac{1}{3}\left(2 d_{i}\right)^{2}-2 d_{i} d_{i}+\frac{2}{3} d_{i}^{2}=0
\end{gathered}
$$

and $d_{i} \leq s_{i}<2 d_{i}$ it follows that

$$
\frac{1}{3} s_{i}^{2}-s_{i} d_{i}+\frac{2}{3} d_{i}^{2} \leq 0 .
$$

Consequently,

$$
\left|Q_{i}\right|>s_{i} d_{i}-\frac{2}{3} d_{i}^{2} \geq \frac{1}{3} s_{i}^{2}=\frac{1}{3}\left|S_{i}\right| .
$$

TheOREM 1. Any sequence of squares homothetic to I with total area greater than or equal to 5 permits an on-line translative covering of I by the method of the movable bottom.

Proof. Let $\left(S_{i}\right)$ be a sequence of homothetic copies of $I$ with total area not smaller than 5 . Assume that $I$ cannot be covered with $S_{1}, S_{2}, \ldots$ by the method of the movable bottom. Obviously, the side length of each square is smaller than 1.

Since $s_{i}<2 d_{i}$ for each positive integer $i$ it follows that the area of $\bigcup \sigma_{i} R_{i} \backslash I$ is smaller than

$$
\frac{1}{2} \cdot 1+\frac{1}{4} \cdot \frac{1}{2}+\frac{1}{8} \cdot \frac{1}{4}+\cdots=\frac{2}{3} .
$$

The sets $Q_{1}, Q_{2}, \ldots$ are pairwise disjoint and contained in $\bigcup \sigma_{i} R_{i}$. This implies that $\sum\left|Q_{i}\right|<1+\frac{2}{3}=\frac{5}{3}$. By Lemma 1 we deduce that $\sum\left|S_{i}\right|<$ $3 \sum\left|Q_{i}\right|<5$, which is a contradiction.

3. The method of the movable bottom and immovable top. Let $S_{i}$ be a homothetic copy of $I$ of side length $s_{i}$, for $i=1,2, \ldots$ The squares of side length smaller than 1 will be divided into two types: basic squares and special squares. Basic squares will be placed according to the method of the movable bottom. Special squares will be placed into

$$
L=\{(x, y) ; x \geq 0,0 \leq y \leq 1\},
$$

side by side, along the top of $L$.

If $s_{1} \geq 1$, then we cover $I$ with $S_{1}$ and stop. Otherwise, the first square is basic. We place $S_{1} \supset R_{1}$ so that

$$
\sigma_{1} R_{1}=\left\{(x, y) ; 0 \leq x \leq d_{1}, 0 \leq y \leq s_{1}\right\} .
$$

By the part of $S_{1}$ used for the covering we mean $Q_{1}=\sigma_{1} R_{1}$. Furthermore, we take $U_{1}=\sigma_{1} R_{1}$. 
Assume that $i>1$ and that the translations $\sigma_{1}, \ldots, \sigma_{i-1}$ have been provided. Moreover, assume that $U_{1}, \ldots, U_{i-1}$ have been defined. If $s_{i} \geq 1$, then we cover $I$ with $S_{i}$ and stop. Assume that $s_{i}<1$.

We find the greatest number $b_{i} \leq 1$ such that each point of $I$ whose $y$-coordinate is smaller than $b_{i}$ belongs to $\bigcup_{j=1}^{i-1} U_{j}$. The set of points of $I$ with $y$-coordinate $b_{i}$ is called the $i$ th bottom. A point of the $i$ th bottom is a surface point if no point of $I$ with the same $x$-coordinate and with a larger $y$-coordinate belongs to $\bigcup_{j=1}^{i-1} U_{j}$.

If $b_{i}<1-s_{i}$, then $S_{i}$ is basic. We place $S_{i} \supset R_{i}$ so that $\sigma_{i} R_{i}$ contains a surface point and that $\sigma_{i} R_{i}$ has the form

$$
\sigma_{i} R_{i}=\left\{(x, y) ; k d_{i} \leq x \leq(k+1) d_{i}, b_{i} \leq y \leq b_{i}+s_{i}\right\},
$$

where $k \in\left\{0,1, \ldots d_{i}^{-1}-1\right\}$. Obviously, $\sigma_{i} R_{i} \subset I$. We take $U_{i}=\sigma_{i} R_{i}$. The part of $S_{i}$ used for the covering is defined as

$$
Q_{i}=\sigma_{i} R_{i} \backslash \bigcup_{j=1}^{i-1} U_{j} .
$$

If $b_{i} \geq 1-s_{i}$, then $S_{i}$ is special. We take $U_{i}=\emptyset$. If $S_{i}$ is the first special square in the sequence, then

$$
\sigma_{i} S_{i}=\left\{(x, y) ; 0 \leq x \leq s_{i}, 1-s_{i} \leq y \leq 1\right\} .
$$

If there is a special square $S_{j}$ with $j<i$, then denote by $k$ the greatest integer smaller than $i$ such that $S_{k}$ is special. Let $t_{k}$ be the number such that

$$
\sigma_{k} S_{k}=\left\{(x, y) ; t_{k}-s_{k} \leq x \leq t_{k}, 1-s_{k} \leq y \leq 1\right\} .
$$

We place $S_{i}$ so that

$$
\sigma_{i} S_{i}=\left\{(x, y) ; t_{k} \leq x \leq t_{k}+s_{i}, 1-s_{i} \leq y \leq 1\right\} .
$$

Obviously,

$$
\sigma_{i} S_{i} \supseteq\left\{(x, y) ; t_{k} \leq x \leq t_{k}+s_{i}, b_{i} \leq y \leq 1\right\} .
$$

This method is called the method of the movable bottom and immovable top. It is easy to see that if the point $(1,1)$ has been covered (with a special square), then $I$ has been covered.

THEOREM 2. The unit square I can be on-line translatively covered with any sequence of homothetic copies of I whose total area is greater than or equal to 4.

Proof. Let $\left(S_{i}\right)$ be a sequence of homothetic copies of $I$ with total area not smaller than 4 . Assume that $I$ cannot be covered with $S_{1}, S_{2}, \ldots$ by the method of the movable bottom and immovable top. Obviously, the side length of each square is smaller than 1 . The parts of the basic squares used for the covering are pairwise disjoint and they are contained in $I$. By Lemma 1 
we deduce that the total area of the basic squares is smaller than $3|I|=3$. The total area of the special squares is smaller than 1 (even if the sequence of squares is infinite). Consequently, $\sum\left|S_{i}\right|<3+1=4$, which is a contradiction.

4. Covering a convex body. We will cover $I$ with squares of side length smaller than $1 / 2$ in the proof of Theorem 3 below.

Lemma 2. Any sequence of squares homothetic to I of side length smaller than or equal to $s$, where $0<s<1$, with total area not smaller than $3+s$ permits an on-line translative covering of $I$.

Proof. Let $S_{i}$ be a homothetic copy of $I$ with a positive homothety ratio not greater that $s$, for $i=1,2, \ldots$ Moreover, let $\sum\left|S_{i}\right| \geq 3+s$. Assume that $I$ cannot be covered with $S_{1}, S_{2}, \ldots$ by the method of the movable bottom and immovable top. The total area of the basic squares is smaller than 3 . The total area of the special squares is smaller than $s$. As a consequence, $\sum\left|S_{i}\right|<3+s$, which is a contradiction.

Theorem 3. Let $C$ be a planar convex body. Any sequence of positive homothetic copies of $C$ with total area not smaller than $14|C|$ permits an on-line translative covering of $C$.

Proof. Let $C_{i}$ be a homothetic copy of $C$ with a ratio $\lambda_{i} \geq 0$, for $i=$ $1,2, \ldots$ Assume that $\sum\left|C_{i}\right| \geq 14|C|$. Obviously, if $\lambda_{i} \geq 1$ for some $i$, then $C$ can be covered with $C_{i}$. Assume that $\lambda_{i}<1$ for $i=1,2, \ldots$

Let $P$ and $T$ be homothetic parallelograms with the homothety ratio 2 such that $P \subset C \subset T$ (see [1]), and let $\mathcal{A}$ be an affine transformation of the plane such that $\mathcal{A}(T)=I$. Denote by $P_{i}$ a homothetic copy of $P$ with the ratio $\lambda_{i}$, for $i=1,2, \ldots$ Each $\mathcal{A}\left(P_{i}\right)$ is a square of side length smaller than 1/2. Furthermore,

$$
\sum\left|\mathcal{A}\left(P_{i}\right)\right| \geq 14|\mathcal{A}(P)|=14 \cdot \frac{1}{4}|\mathcal{A}(T)|=3+\frac{1}{2} .
$$

By Lemma 2 , the sequence $\left(\mathcal{A}\left(P_{i}\right)\right)$ permits an on-line translative covering of $I$. Since $\mathcal{A}\left(P_{i}\right) \subset \mathcal{A}\left(C_{i}\right)$ it follows that $\left(\mathcal{A}\left(C_{i}\right)\right)$ permits an on-line translative covering of $I=\mathcal{A}(T) \supset \mathcal{A}(C)$. Consequently, $\left(C_{i}\right)$ permits an on-line translative covering of $C$.

\section{References}

[1] H. Groemer, Covering and packing by sequences of convex sets, in: Discrete Geometry and Convexity, Ann. New York Acad. Sci. 440, 1985, 262-278.

[2] B. Grünbaum, Measures of symmetry of convex sets, in: Convexity, Proc. Sympos. Pure Math. 7, Amer. Math. Soc., Providence, 1963, 233-270. 
[3] J. Januszewski and M. Lassak, On-line covering the unit cube by cubes, Discrete Comput. Geom. 12 (1994), 433-438.

[4] -, -, On-line covering the unit square by squares and the three-dimensional unit cube by cubes, Demonstratio Math. 28 (1995), 143-149.

[5] J. Januszewski, M. Lassak, G. Rote and G. Woeginger, On-line q-adic covering by the method of the $n$-th segment and its application to on-line covering by cubes, Beiträge Algebra Geom. 37 (1996), 51-65.

[6] W. Kuperberg, On-line covering a cube by a sequence of cubes, Discrete Comput. Geom. 12 (1994), 83-90.

[7] M. Lassak, A survey of algorithms for on-line packing and covering by sequences of convex bodies, Bolyai Soc. Math. Stud. 6, János Bolyai Math. Soc., Budapest, 1997, $129-157$.

[8] J. W. Moon and L. Moser, Some packing and covering theorems, Colloq. Math. 17 (1967), 103-110.

Janusz Januszewski

Institute of Mathematics and Physics

University of Technology and Life Sciences

Kaliskiego 7

85-796 Bydgoszcz, Poland

E-mail: januszew@utp.edu.pl

Received December 19, 2008;

received in final form March 24, 2009 